\title{
MAPEAMENTO DA QUALIDADE DA ÁGUA SUBTERRÂNEA RASA ATRAVÉS DE ÍNDICE FISICO-QUÍMICO EM CAMPOS DOS GOYTACAZES, RJ.
}

\author{
Cleber de Jesus Santos $^{(a)}$, Eduardo Bulhões ${ }^{(b)}$ \\ (a) Mestrando. Laboratório de Geografia Física. Programa de Pós-Graduação em Geografia. Instituto de Ciências da \\ Sociedade e Desenvolvimento Regional. Universidade Federal Fluminense \\ cleber.geofisico@gmail.com \\ (b) Prof. Adjunto. Laboratório de Geografia Física. Programa de Pós-Graduação em Geografia. Instituto de Ciências \\ da Sociedade e Desenvolvimento Regional. Universidade Federal Fluminense \\ eduardobulhoes@id.uff.br
}

\section{EIXO: BACIAS HIDROGRÁFICAS E RECURSOS HÍDRICOS, ANÁLISE, PLANEJAMENTO E GESTÃO}

\begin{abstract}
Resumo
O artigo apresenta o mapeamento da qualidade da água subterrânea em toda a extensão do município de Campos dos Goytacazes para o ano de 2015. A partir da construção do índice físico-químico IQA identificou-se a qualidade dos poços, índice composto do produto ponderado de dez parâmetros (ph, $\mathrm{Fe}, \mathrm{Mn} . \mathrm{Na}, \mathrm{Ca}, \mathrm{Cl}, \mathrm{Mg}, \mathrm{S}, \mathrm{Cu}, \mathrm{Zn}$ ), calculados em 164 poços de profundidade entre 8 e $14 \mathrm{~m}$. Posteriormente, elaboraram-se um mapa contendo o mapa auxiliar ampliado correspondente ao setor central do município, cujos dados estiveram um maior adensamento. A interpretação do mapa considerou como principal fator de influência as interações socioambientais, visto que se trata de aquífero livre cujo nível estático encontra-se a baixas profundidades. O presente artigo configura-se num valioso método de pesquisa, seja pontual ou espacial, para identificação dos padrões característicos de qualidade da água.
\end{abstract}

Palavras chave: Hidrogeologia; Hidrogeoquímica; Potabilidade.

\section{Introdução}

Os desafios concernentes a gestão sustentável da água no mundo atual implica num adequado monitoramento da qualidade deste recurso face aos problemas resultantes do crescente e indiscriminado uso. Do ponto de vista hidrogeológico a qualidade é tão importante quanto a quantidade, visto que a disponibilidade dos recursos hídricos para determinados usos depende fundamentalmente da característica físico-química, biológica e radiológica das águas (SANTOS, 1997).

Nessa perspectiva, o presente estudo faz-se oportuno, pois propõe uma metodologia funcional para avaliar a qualidade da água subterrânea em 2015, na forma de caracterização seguida de espacialização. Esta pesquisa objetiva detectar o panorama da qualidade da água no município de Campos dos Goytacazes - 
$\mathrm{RJ}$, visando ampliar os conhecimentos hidrogeológicos e hidrogeoquímicos da área de forma a orientar pesquisas futuras, frente a atual escassez de dados desta natureza no município. Para tal, foi elaborado um índice de qualidade da água com base em parâmetros físico-químicos contidos nos relatórios de 164 poços de profundidade variando entre 8 e $14 \mathrm{~m}$. O índice calculado será espacializado, originando mapas referentes aos três anos especificados. Diante do mapa será feito análises individuais e comparativas, estabelecendo qualificação de domínios, correlações com a geologia local e influência antrópica.

\section{Caracterização da área de estudo}

O município de Campos dos Goytacazes pertence à mesorregião Norte Fluminense do estado do Rio de Janeiro, Brasil, possuindo uma área de 4.026,696 km² - o maior do estado (Figura 01).

Figura 1: Campos dos Goytacazes - RJ.

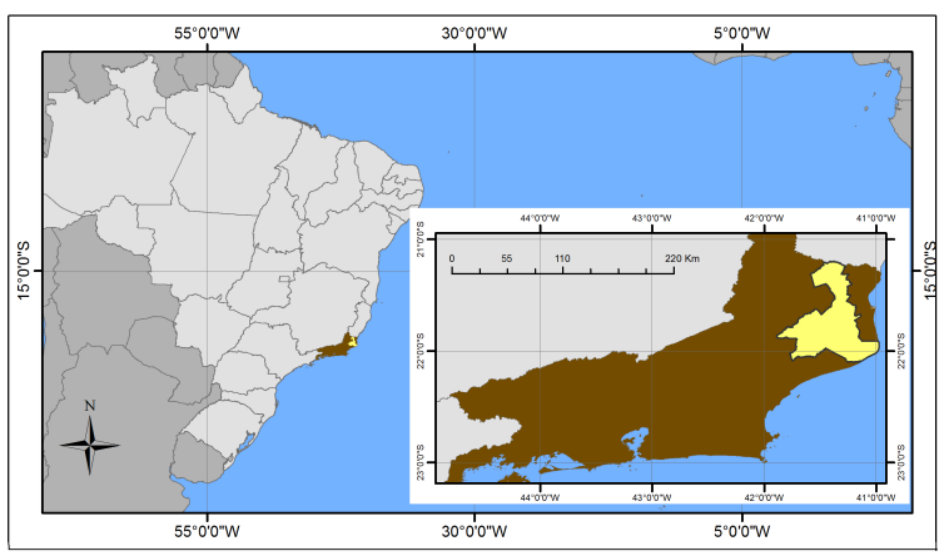

Geomorfologicamente, possui unidades como a planície fluvial e marinha (36,66\% em área), tabuleiros originados dos depósitos terciários da Formação Barreiras $(11,4 \%)$, colinas suave-onduladas ou mar de morro pertencente ao embasamento cristalino $(37,44 \%)$ e escarpas de serras $(12,4 \%)$ (Ramalho, 2005). O município está inserido no contexto geológico da bacia de campos contendo uma unidade sedimentar do Fanerozóico composta por sedimentos do quaternário fluvio-lagunar (argilas e siltes de planície de inundação), do quaternário costeiro (areias quartzosas), e do terciário, representado pela Formação Barreiras (sedimentos continentais arenosos e argilosos) (Ferrari et. al., 1981) (Figura 02). Terrenos sedimentares que justificam a ocorrência dos principais aquíferos da área em questão. O Aquífero Fluviodeltaico, localizado na margem sul do Rio Paraíba Sul, próximo a cidade de Campos dos Goytacazes, é um aquífero livre cuja espessura dos sedimentos possui $90 \mathrm{~m}$ com intercalações de argilas. O Aquífero Emborê, localizado nos arredores do Farol de São Tomé, possui pacotes confinados de sedimentos 


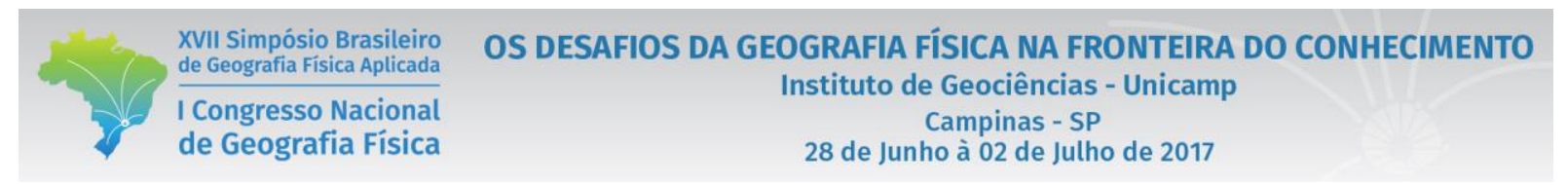

arenosos intercalados por argilas, níveis conchíferos e presença de madeira fóssil. Este com espessura média de 200m. O Aquífero São Tomé tem dois compartimentos distintos contendo águas ferruginosas. Por fim o Aquífero Barreiras, livre e de baixa produtividade (Albuquerque e Oliveira, 1999). Os poços utilizados nesta pesquisa, em sua maioria, captam água do aquífero fluvio-deltaico.

Figura 2: Mapa Geológico de Campos dos Goytacazes.

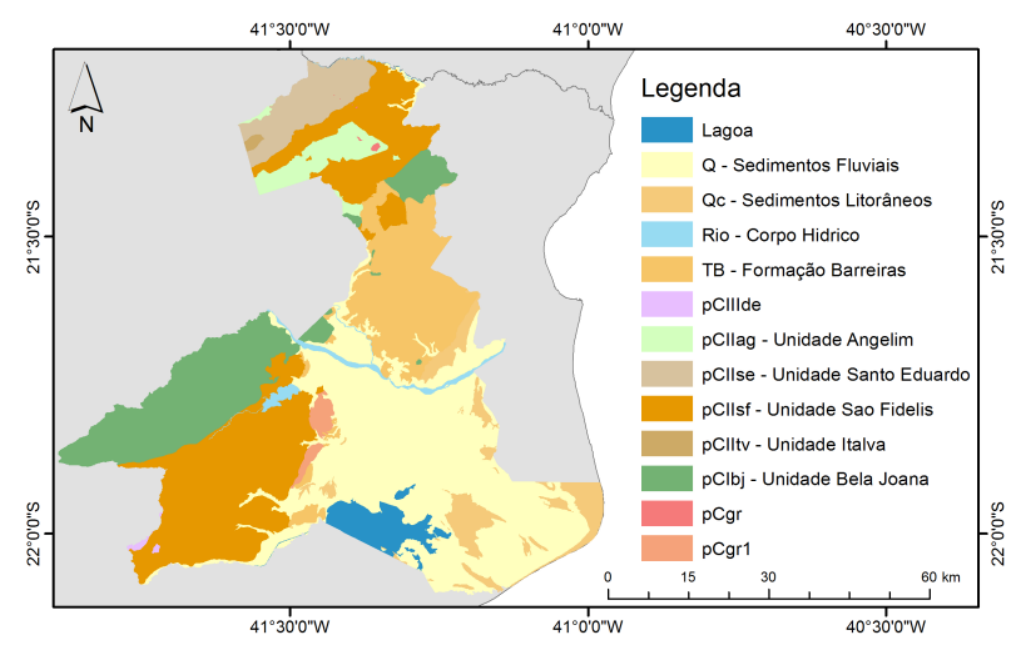

\section{Metodologia}

A pesquisa prescindiu inicialmente da coleta de informações bibliográficas sobre a área em questão seguido da coleta de dados de análise poços rasos que, por sua vez, foram obtidos através da FUNDENOR (Fundação Norte Fluminense de Desenvolvimento Regional). Na sequência, a partir dos parâmetros físicoquímicos contidos nos relatórios de poço, desenvolvemos um índice de qualidade físico-químico. O IQA é calculado pelo produto ponderado das qualidades de água correspondentes às variáveis que integram o índice (Tabela I).

Tabela I - Fórmula do índice de qualidade fisico-químico.

\begin{tabular}{|lc|}
\hline Legenda: & IQA $=\prod_{\mathrm{i}=1}^{\mathrm{n}} \cdot \mathrm{q}_{\mathrm{i}}{ }^{\text {wi }}$ \\
n: número de parâmetros & qualidade em cada parâmetro. \\
wi: peso referente a cada parâmetro. & $0<\mathrm{IQA}<100$ \\
IQA: qualidade relativa a cada poço. & \\
\hline
\end{tabular}

A variável $q$ é calculada para cada parâmetro, sejam eles: ph, $\mathrm{Fe}, \mathrm{Mn}, \mathrm{Na}, \mathrm{Ca}, \mathrm{Cl}, \mathrm{Mg}, \mathrm{S}, \mathrm{Cu}, \mathrm{Zn}$. O cálculo é fundamentado na Portaria 2.914/2011 do Ministério da saúde, valores de referência de cada

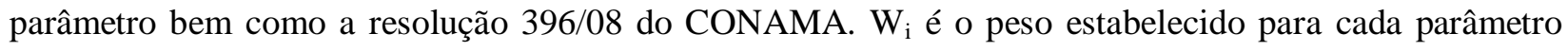
tendo como referência a variabilidade envolvida e a vulnerabilidade embutida em cada parâmetro. $\mathrm{O}$ índice de qualidade (IQA) calculado para todos os poços foi utilizado na confecção dos mapas através do 
XVII Simpósio Brasileiro de Geografia Fisica Aplicada

I Congresso Nacional de Geografia Física

\section{OS DESAFIOS DA GEOGRAFIA FÍSICA NA FRONTEIRA DO CONHECIMENTO \\ Instituto de Geociências - Unicamp \\ Campinas - SP \\ 28 de Junho à 02 de Julho de 2017}

software SURFER 11 da Golden Software Inc (Figura 03 e 04). Os mapas de localização da área, uso e cobertura do solo (Figura 01) e geológico (Figura 02) foram produzidos no software ARCGIS 10.

\section{Resultados}

O mapa de qualidade físico-química (Figura 03) obtido apresenta um adensamento das medidas no perímetro urbano, naturalmente, em virtude da oferta desigual destes dados no espaço. A disposição destes, associado ao fato de serem poços rasos, em um substrato eminentemente arenoso, constitui um contexto de alto grau de vulnerabilidade a que este recurso hídrico está sujeito.

Figura 03: Mapa de Qualidade Físico-química da água subterrânea em Campos dos Goytacazes em 2015.

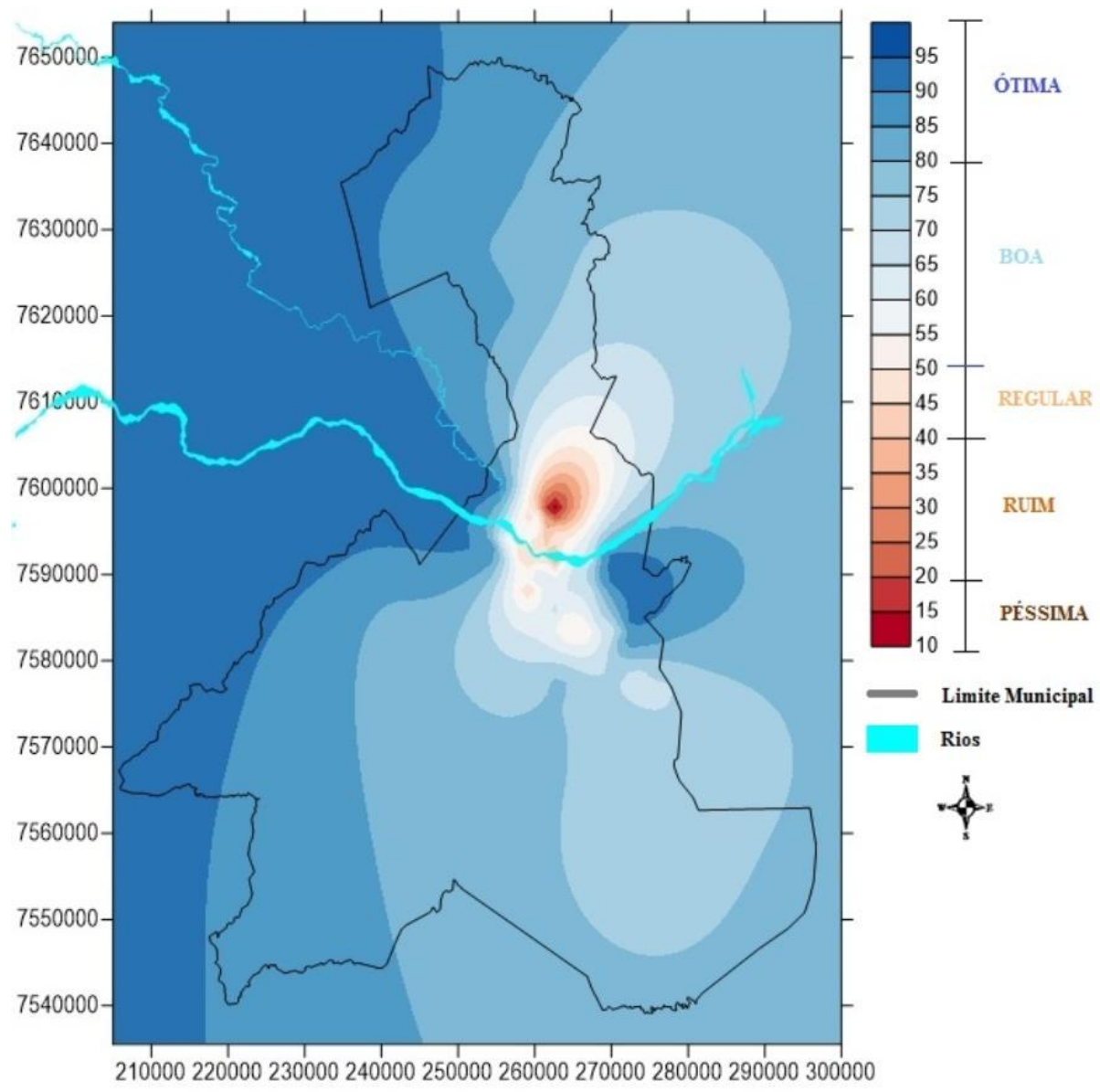

A presença de feições em vermelho no mapa (Figura 03) é marcante no compartimento norte da zona urbana, sinalizando um padrão característico de alteração danosa. A referida feição de baixa qualidade associa-se diretamente à ação antrópica na forma de passivo ambiental. Possivelmente relacionada aos 


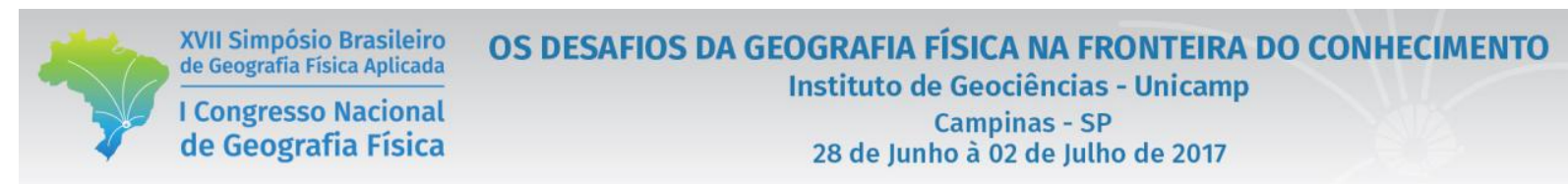

efeitos do lixão desativado que, por mais de vinte anos, funcionou neste setor da zona urbana, comprometendo o lençol freático das imediações (Coelho et. al, 2002).

No âmbito geral existem dois domínios característicos, sendo um de boa a ótima qualidade correspondente a alguns pontos do setor sudoeste e norte, contendo distritos rurais no qual a concentração populacional é reduzida. O segundo domínio, ampliado no mapa (Figura 03), correspondente ao setor central, possui diversos pontos cuja qualidade vai de regular a ruim, característicos de alterações das águas superficiais.

Figura 04: Mapa de Qualidade Físico-química do perímetro urbano.

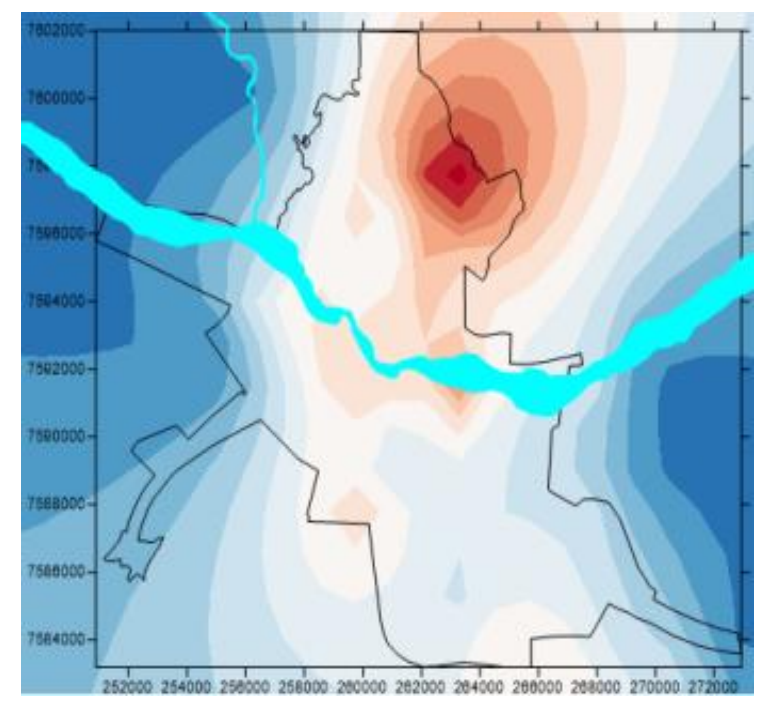

Alguns parâmetros físico-químicos apresentaram considerável influência nos valores de IQA, sendo que o ferro $(\mathrm{Fe})$ e o ph (potencial hidrogeniônico), em uma quantidade considerável dos dados, apresentou divergência quanto ao valor máximo permitido (VMP) estabelecidos em leis atualmente em vigor no país. O fator hidrogeoquímico, proveniente da interação solo/água/rocha, responde por esta característica visto que os sedimentos do Grupo Barreiras, marcante no município, apresenta-se na forma de sedimentos detríticos intemperizados, contendo argilas de características ferruginosas (Morais, et. al, 2006).

\section{Conclusões}

1. O uso da água subterrânea pela população de Campos dos Goytacazes é uma realidade que carece de monitoramento tanto pela conservação deste bem quanto pela preservação da saúde daqueles que dela se utiliza. A oferta abundante deste recurso, em profundidades relativamente baixas somado às características do substrato rochoso de favorabilidade a captação, aliado a problemas pretéritos de abastecimento residencial, responde por esta demanda crescente no município. 
2. O índice físico-químico desenvolvido configura-se como uma metodologia funcional para atribuição qualitativa a partir de dados quantitativos, refletindo as informações contidas nos relatórios de forma conjunta. Consequentemente, a disposição espacial deste possibilitou identificar estados de alteração da água subterrânea rasa de forma clara e segura. Metodologia esta que naturalmente pode ser aplicada na observação de quaisquer fenômenos dependentes de um número considerável de parâmetros.

3. O nível de análise estabelecido possibilitou o entendimento do panorama municipal da qualidade da água subterrânea em profundidades rasas, tendo como base a análise físico-química de 164 poços no ano de 2015. A partir dos resultados em mapa entendemos que a vulnerabilidade intrínseca destas águas está diretamente relacionada à ação antrópica, principalmente nas concentrações urbanas.

4. No mapa detectamos que as principais alterações na qualidade da água ocorreram no setor central da cidade (perímetro urbano). Os baixos valores de IQA estão localizados no distrito de Guarus. Nesse, as condições deficitárias de saneamento básico, a deposição inadequada de resíduos sólidos em determinados locais bem como a proximidade com lagoas contaminadas por dejetos de esgoto podem estar relacionadas a esses baixos índices.

5. O presente estudo obteve sucesso na medida em que identifica padrões característicos de qualidade da água em toda extensão do município; oferece subsídios para estudos de remediação em aquíferos degradados; apresenta-se como estímulo a novas pesquisas desta natureza; e colabora para o entendimento do estado atual dos corpos hídricos subterrâneos, auxiliando diretamente na promoção de políticas públicas de preservação ambiental e de saúde pública no município de Campos dos Goytacazes.

\section{Agradecimentos}

A FUNDENOR (Fundação Norte Fluminense de Desenvolvimento Regional) pela produção e disponibilização dos relatórios de análise físico-químicos. Especialmente na figura de Lauro Robaina Rangel, Engenheiro Agrônomo, responsável pelo laboratório de análise físico-química.

\section{Bibliografia}

ALBUQUERQUE, H.J.T.R, OLIVEIRA, J.E.C. A Importância das Águas Subterrâneas. Revista Abastece. Ano I, $\mathrm{N}^{\circ}$ 4. 1999.

COELHO, M.G; LIMA, S.C; MARAGNO, A.L.F; ALBUQUERQUE, Y.T; LEMOS, J.C; SANTOS, C.L. dos; BRANDÃO, S.L. 2002. Contaminação das Águas do Lençol Freático por Disposição Inadequada de Resíduos Sólidos Urbanos em Uberlândia-MG/Brasil. Congresso Interamericano de Engenharia Sanitária e Ambiental, XXVIII, 2002, Cancun. 
FERRARI, A. L.; MELO, E. F.; VAZ, M. A. A.; DALCOMO, M. T.; BRENNER, T. L.; SILVA, V. P. \& Nassar, W.M. 1981. Projeto Carta Geológica do Rio de Janeiro - Bloco Campos - In: volume I - DRM - Geomitec, Geologia e Mineração Trabalhos Técnicos Ltda. Rio de Janeiro. 172p. Relatório Técnico.

MORAIS, R.M.O. de; MELLO, C.L; COSTA, F.O; SANTOS, P.F. 2006. Fácies Sedimentares e Ambientes Deposicionais Associados aos Depósitos da Formação Barreiras no Estado do Rio de Janeiro. Revista do Instituto de Geociências - USP. V. 6, No 2, 2006.

OLIVEIRA, L.I. de; LOUREIRO, C.O. 1998. Contaminação de Aquíferos por Combustíveis Orgânicos em Belo Horizonte: avaliação preliminar. Congresso Brasileiro de Águas Subterrâneas, X, 1998, São Paulo.

RAMALHO, R.S. Diagnóstico do meio físico como contribuição ao planejamento do uso da terra do município de Campos dos Goytacazes. 2005. 123p. Tese (Doutorado em Ecologia e Recursos Naturais).

Programa de Pós-Graduação em Ecologia e Recursos Naturais, Universidade Estadual do Norte Fluminense. Centro de Biociências Biotecnologia - Campos dos Goytacazes, RJ. 2005.

SANTOS, A. C. Noções de hidroquímica. In: Hidrogeologia: conceitos e aplicações. 1 ${ }^{\mathrm{a}}$ Ed. Fortaleza; CPRM LABHID - UFPE, 1997. p. 81-108. 